\title{
Primary Biliary Cholangitis and Sjogren's Syndrome with Unspecified Neuropathy: A Case Report
}

\author{
Iquebal Hasan ${ }^{1 *}$, Monique Duru ${ }^{2}$, Jamie Bloss ${ }^{3}$ and Amna Hasan ${ }^{4}$ \\ ${ }^{1}$ Clinical Assistant Professor, Oral Medicine, ECU School of Dental Medicine, USA \\ ${ }^{2} D 3$ Student, ECU School of Dental Medicine, USA \\ ${ }^{3}$ Liaison Librarian, Allied Health Sciences and Dental Medicine, East Carolina University, USA \\ ${ }^{4}$ Clinical Assistant Professor, Orofacial Pain, ECU School of Dental Medicine, USA
}

*Corresponding author: Iquebal Hasan, Clinical Assistant Professor, Oral Medicine,

ECU School of Dental Medicine, USA.

Received Date: August 20, 2020

Published Date: Septermber 17, 2020

\begin{abstract}
We describe a unique and rare presentation of numbness in the oral cavity due to undiagnosed Sjogren's Syndrome. Accompanied with primary biliary cholangitis, these syndromes have a limited presence within oral related cases as shown by literary searches. This is one of the few cases reported demonstrating Sjogren's Syndrome and primary biliary cholangitis displaying paresthesia in the oral cavity, dysphagia, and ascites.

Sjogren's Syndrome and primary biliary cholangitis are two complex and chronic autoimmune diseases that can coexist and can present in a variety of ways. Sjogren's syndrome has a major effect on exocrine glands, such as the salivary gland. This will consequently result in oral dryness that can cause dysphagia. Sjogren's syndrome has multiple forms, being primary and secondary. Secondary Sjogren's Syndrome is when patient has previously been diagnosed with another autoimmune disease presenting before the diagnosis of Sjogren's. In rare cases patients with Sjogren's Syndrome may develop primary biliary cholangitis. We report a case of primary biliary cholangitis and Sjogren's Syndrome, presenting with paresthesia of the perioral region and dysphagia. After thorough literature searches, there proves to be very few similar cases in the dental literature $[1,2]$.
\end{abstract}

\section{Case Report}

A female was referred to East Carolina University School of Dental Medicine for evaluation of a sudden onset of tingling and numbness of the upper lip, maxillary and mandibular gingiva and the tip of the tongue. Her medical history included a recent diagnosis of primary biliary cholangitis and she presented with her first episode of ascites. She informed that she was scheduled to get the drainage of fluid done the next day. Extraoral examination also confirmed numbness presentation upon palpation of both sides of her face with patient's eyes closed. Her other symptoms like dry mouth, dry eyes, fatigue and body aches were consistent with Sjogren's Syndrome. It was decided to order blood work for diagnosis of SS. After a consultation with Orofacial Pain we also decided on an MRI of the brain and skull, with and without contrast for evaluation of cranial nerves V and VII. The MRI demonstrated unremarkable imagining of the brain and no compression or abnormality of the Vth and VIIth cranial nerves. The blood work resulted in a positive diagnosis for Sjogren's Syndrome. Sjogren's Syndrome can cause different types of neuropathies and it was agreed that her tingling and numbness has most likely been caused by SS. It was decided to refer the patient to rheumatologist for further evaluation and proper treatment. 


\section{Results}

MRI of the brain and skull base had findings of a small vessel near the cisternal portion of the right fifth cranial nerve, however there was no compression or definite abnormality. The ordered items for blood work were Sjogren's Ab, Anti-SS-A/-SS-B, CCP Antibodies IgG/IgA, ANA with Reflex, Sedimentation RateWestergren, and C-Reactive Protein. Sjogren's Anti-SS-A resulted in a high reading of 1.2. ANA with Reflex Antinuclear Antibodies resulted in an extremely positive reading of 1:1280 with a speckled pattern. Sedimentation Rate-Westergren resulted in a reading of 33 $\mathrm{mm} / \mathrm{hr}$ and C-Reactive Protein resulted in a reading of $7 \mathrm{mg} / \mathrm{L}$.

\section{Discussion}

The diagnosis of sjogren's syndrome was confirmed by the blood work displaying both a highly positive antibody count and the specific speckled pattern. The ANA with Reflex Antinuclear Antibodies results were showed an exceptionally positive reading with a speckled pattern, which indicates Sjogren's Syndrome. With more awareness of these presentations and symptoms in dentistry, we hope to reduce the diagnosis time for patients. Eventually this will help in improving their quality of life through treatment Table 1.

Table 1: Lab Results.

\begin{tabular}{|c|c|c|c|}
\hline Tests & Results & Flag & Units \\
\hline Sjogren's Ab Anti-SS-A & (Anti-SS-A): 1.2 & High & $\mathrm{AI}$ \\
\hline Sjogren's Ab Anti-SS-B & (Anti-SS-B): $<0.2$ & & \\
\hline CCP Antibodies IgG/IgA & 7 & & \\
\hline ANA w/Reflex Antinuclear Antibodies, IFA & $1: 1280$ & HIGH; Positive abnormal & \\
\hline Sedimentation Rate-Westergren & 33 & & $\mathrm{~mm} / \mathrm{hr}$ \\
\hline C-Reactive Protein, Quan & 7 & $\mathrm{mg} / \mathrm{L}$ \\
\hline
\end{tabular}

\section{Conclusion}

There is limited literature in dentistry which talks about Sjogren's Syndrome and primary biliary cholangitis occurring simultaneously. Patient's with these two conditions, presenting with facial neuropathy is rare and unreported to the best of our knowledge. We are hoping that this case report will be able to increase awareness of possible presenting symptoms of these conditions. This will reduce the chances of patients being subjected to long periods of suffering due to lack of diagnosis.

\section{Conflict of Interest}

No conflict of interest.

\section{References}

1. Lins L, Paraná R, Reis S, Pereira Falcão A (2014). Primary Biliary Cirrhosis and Primary Sjögren's Syndrome: Insights for the Stomatologist. Case Rep Gastroenterol 8(2): 251-256.

2. Mandel L, Dehlinger N (2003) Primary biliary cirrhosis and Sjögren's syndrome: case report. Journal of Oral and Maxillofacial Surgery 61(11): $1358-1361$.

\section{Acknowledgement}

None. 